\title{
Demonstration of a geometric isomer of bilirubin-IX $\alpha$ in the serum of a hyperbilirubinaemic newborn infant and the mechanism of jaundice phototherapy
}

\author{
Shoju ONISHI, Kenichi ISOBE, Susumu ITOH, Noboru KAWADE and Satoru SUGIYAMA \\ Department of Pediatrics, Nagoya City University Medical School, Kawasumi Mizuho-ku, Nagoya, Japan
}

\author{
(Received 6 February 1980)
}

\begin{abstract}
Photobilirubin-IX $\alpha$ in the serum of a hyperbilirubinaemic newborn infant was demonstrated for the first time by reversed-phase high-pressure liquid chromatography. The concentrations increased significantly during phototherapy. Corresponding to these changes in the serum, biliary unconjugated bilirubin concentrations increased markedly but photobilirubin-IX $\alpha$ was not detected in the bile. Moreover, large amounts of 'unknown pigment' that are diazo-negative appeared in the bile during phototherapy.
\end{abstract}

Phototherapy is an established procedure in neonatal medicine (Seligman, 1977). Despite its extensive use in newborn infants with hyperbilirubinaemia, the complete mechanism is not known (McDonagh \& Ramonas, 1978). However, it was observed that the considerable amounts of unconjugated bilirubin appear in the bile of Gunn rat (Ostrow, 1971; Ostrow et al., 1974) and newborn infants (Lund \& Jacobsen, 1972) after irradiation with blue light and that bilirubin-IX $\alpha$ is normally in the $Z Z$-configuration at the $\mathrm{C}-5$ and $\mathrm{C}-15$ bridges (Bonnett et al., 1976, 1978) and undergoes rapid and reversible photoisomerization on irradiation with blue light, giving a photobilirubin with $E$ configuration (McDonagh et al., 1979). The present view of the predominant pathways of removal of bilirubin from the body in the course of phototherapy is photoisomerization (Lightner et al., 1979a,b; Stoll et al., 1979; Onishi et al., 1979a,b). Chromatography and purification of geometric isomers of naturally occurring bilirubin-IX $\alpha$ have been frustrated by its tendency to revert to $(Z Z)$ bilirubin-IX $\alpha$. However, we were able to resolve bilirubin-IX $\alpha$ into its components by h.p.l.c. (Onishi et al., 1979a,b, 1980c). This method was applied to the analysis of biological samples to provide insight into the mechanism of phototherapy and in the present paper we demonstrate the increase of photobilirubin in serum with considerable increases in biliary excretion of ( $Z Z)$-bilirubin-IX $(x$ and the appearance of an unknown pigment during phototherapy. A preliminary report of these results has been published previously (Onishi et al., 1979a,b).

Abbreviation used: h.p.l.c., high-pressure liquid chromatography.
The predominant natural bilirubin isomer, $(Z Z)$ bilirubin-IX $\alpha$, will often be abbreviated by the term bilirubin. Mention of the $E$-configuration refers to any of the photoisomers $(E Z, Z E$ or $E E)$.

\section{Materials and methods}

H.p.l.c.

Equipment, operation, preparation of calibration curves and peak assignments were as described previously (Onishi et al., 1980c).

The tests on thermal and catalytic reversion of photobilirubin to $(Z Z)$-bilirubin-IX $\alpha$ were performed as described previously (Onishi et al., $1980 c$ ).

Serum, urine and bile sampling. Bile and serum samples were obtained from duodenal aspiration and heel or vein puncture respectively before and during phototherapy. Bile and urine samples were either immediately analysed or stored at $-70^{\circ} \mathrm{C}$ in the dark until analysis, and serum samples were stored at $-20^{\circ} \mathrm{C}$ in the dark until analysis (within 1 week). It was confirmed that such storage did not affect significantly the results. Informed consent was obtained in all cases.

Sample preparation for h.p.l.c. Acetonitrile (3vol.; $60 \%, \mathrm{v} / \mathrm{v}$ ) in $0.01 \mathrm{M}$-phosphate buffer (pH 8.0) was added to 5 vol. of the bile, which was vortex-mixed for $30 \mathrm{~s}$ and then centrifuged for $5 \mathrm{~min}$ at $1000 \mathrm{rev} . / \mathrm{min}$. For the serum sample, the same procedure was used as for the bile except that $4 \mathrm{vol}$. of $60 \%(\mathrm{v} / \mathrm{v})$ acetonitrile in $0.01 \mathrm{M}$-phosphate buffer (pH8.0) was added to 1 vol. of the serum. Of the supernatant $10-100 \mu \mathrm{l}$ was applied on the chromatograph. 


\section{Pigments and reagents}

Bilirubin (E. Merck, Darmstadt, Germany) and tetra-n-butylammonium hydroxide (Wako, Osaka, Japan) were used without further purification. Pentan-2-one (Tokyo Kasei, Tokyo, Japan) was distilled before use. Acetonitrile and all other reagents used were of analytical grade.

\section{Results and discussion}

\section{Effect of phototherapy on the serum photobilirubin} levels

By using reversed-phase h.p.l.c. for photobilirubin analysis as described previously (Onishi et al., $1979 a, b)$, we analysed the bile pigments in the serum of a hyperbilirubinaemic newborn infant before and during phototherapy. In addition to the large peak of bilirubin with retention time of $116 \mathrm{~min}$, one prominent peak with a retention time of $85 \mathrm{~min}$ was observed, indicating that the peak is a more polar bilirubin than bilirubin (Fig. 1). The fact that photobilirubin is the IX $\alpha$-isomer was also confirmed by the following observation. Azodipyrroles derived from photobilirubin consisted of equal amounts of endo- and exo-vinyl isomers. This was proved by azopigment analysis on h.p.l.c. as described previously (Onishi et al., 1979a, 1980a,b). The more polar bilirubin peak was collected and kept at $50^{\circ} \mathrm{C}$ for $30 \mathrm{~min}$ in the dark or with addition of a trace of trifluoroacetic acid (Lightner et al., $1979 a$ ) and then analysed again by h.p.l.c. in the same conditions. The peak height of bilirubin was increased, corresponding to the disappearance of the peak at the retention time of the polar bilirubin. A

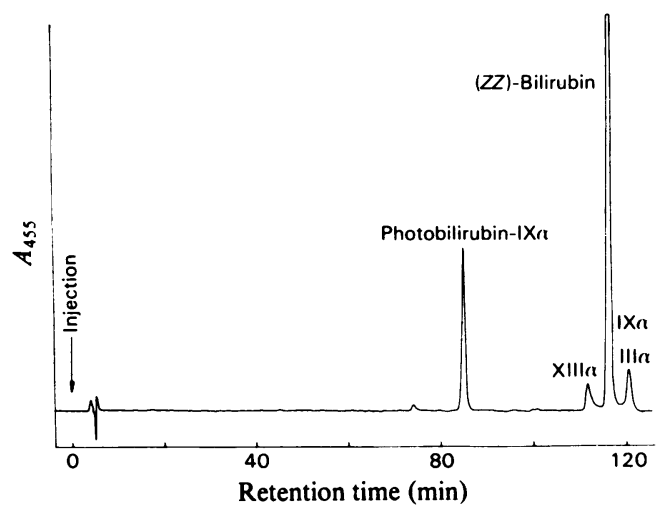

Fig. 1. H.p.l.c. scan of the serum of hyperbilirubinaemic infants during phototherapy

One peak of a more polar bilirubin-IXa (photobilirubin-IX $(\alpha)$ than (ZZ)-bilirubin-IX $\alpha$ was observed to increase markedly during phototherapy. See the text for further details of the analytical conditions. photoinduced $Z \rightarrow E$ transition in bilirubin has been observed by h.p.l.c. (Onishi et al., 1979a,b; McDonagh et al., 1979), solvent solubility (Stoll et al., 1979) and difference spectrophotometry (Lightner et al., 1979a,b). Bilirubin usually exists as an intramolecularly hydrogen-bonded structure, which accounts for its near insolubility in water at physiological pH (Bonnett et al., 1978). Molecular models show that such extensive hydrogen-bonding is sterically impossible for the three $E$-configurational isomers $(E Z, Z E$ and $E E)$. Consequently compounds in the $E$-configuration are more hydrophilic and thermodynamically less stable than

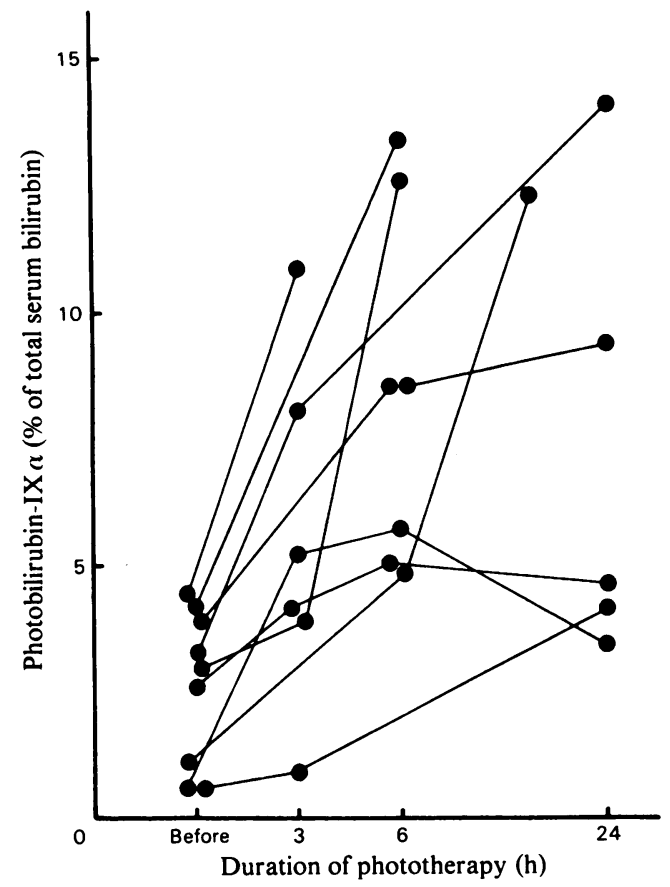

Fig. 2. Effect of phototherapy on photobilirubin-IXa as a percentage of total serum bilirubin concentration

Table 1. Change in photobilirubin-IXa concentration as a percentage of total serum bilirubin during phototherapy

Change in photobilirubin-IX $\alpha(\%)$

\begin{tabular}{lcccc}
\cline { 3 - 4 } $\begin{array}{c}\text { Before } \\
\text { phototherapy }\end{array}$ & \multicolumn{3}{c}{$\begin{array}{c}\text { Duration } \\
\text { of phototherapy (h) }\end{array}$} \\
Mean & 2.6 & 5.5 & 8.3 & 7.9 \\
S.D. & 1.4 & 3.2 & 3.2 & 4.2 \\
Number of patients & 9 & 6 & 7 & 6
\end{tabular}


bilirubin and revert readily to bilirubin in thermal, catalytic and photochemical conditions (McDonagh et al., 1979; Onishi et al., 1979b). Accordingly it was concluded that the more polar bilirubin on h.p.l.c. of the serum is photobilirubin. Fig. 2 shows the changes in the percentage of photobilirubin-IX $\alpha$ of total serum bilirubin concentration during phototherapy. The significant average increment in photobilirubin from 2.6 to $8.3 \%$ occurred within several hours of initiation of blue-light irradiation (Table 1). A similar observation that photobilirubin was generated in the serum from irradiated jaundiced Gunn rats has been made from spectroscopic measurements (L. A. Palma \& A. F. McDonagh, personal communication). However, they have been unable to separate photobilirubin from the serum and identify it chromatographically owing to its thermal reconversion into bilirubin during isolation.
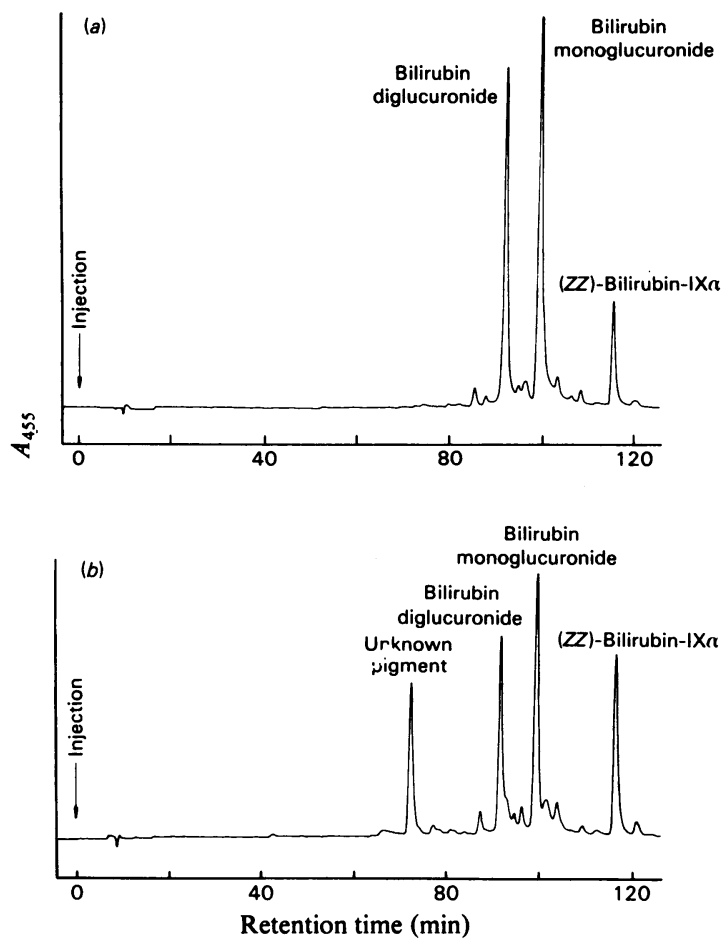

Fig. 3. H.p.l.c. scans of the bile obtained from the duodenal aspiration before and during phototherapy H.p.l.c. scans of the bile are shown just before phototherapy $(a)$ and at $3 \mathrm{~h}$ after phototherapy $(b)$. Unlike the h.p.l.c. conditions for conjugated-bilirubin analysis as described previously (Onishi et al., $1980 b$ ), two peaks of endo-vinyl and exo-vinyl monoglucuronide isomers are not separable in these separation conditions, for which the reason is not known.
Effect of phototherapy on the biliary and urinary excretion of bile pigments

Scanning by h.p.l.c. of bile pigments in newborn-infant duodenal bile before and during phototherapy is shown in Figs. $3(a)-3(b)$. The photobilirubin peak was not detected on h.p.l.c. scans. Bilirubin-IX $\alpha$ mono- and di-glucuronide peaks were decreased, but the peak of unconjugated bilirubinIX $\alpha$ significantly increased during phototherapy. These peaks were identified by azopigment analysis on h.p.l.c. (Onishi et al., 1979a, 1980a,b). Moreover an 'unknown-pigment' peak, which was diazo-negative, with a retention time of $70 \mathrm{~min}$ appeared in the bile during phototherapy (Figs. $3 a$ and $3 b$ ). The concentrations of ( $Z Z)$-bilirubin-IX $\alpha$ and of the unknown pigment increased considerably in bile (Fig. 4), similar but less pronounced changes being found for urine (Fig. 5). The 'unknown pigment' in the urine on h.p.l.c. may correspond to the $415 \mathrm{~nm}$ -
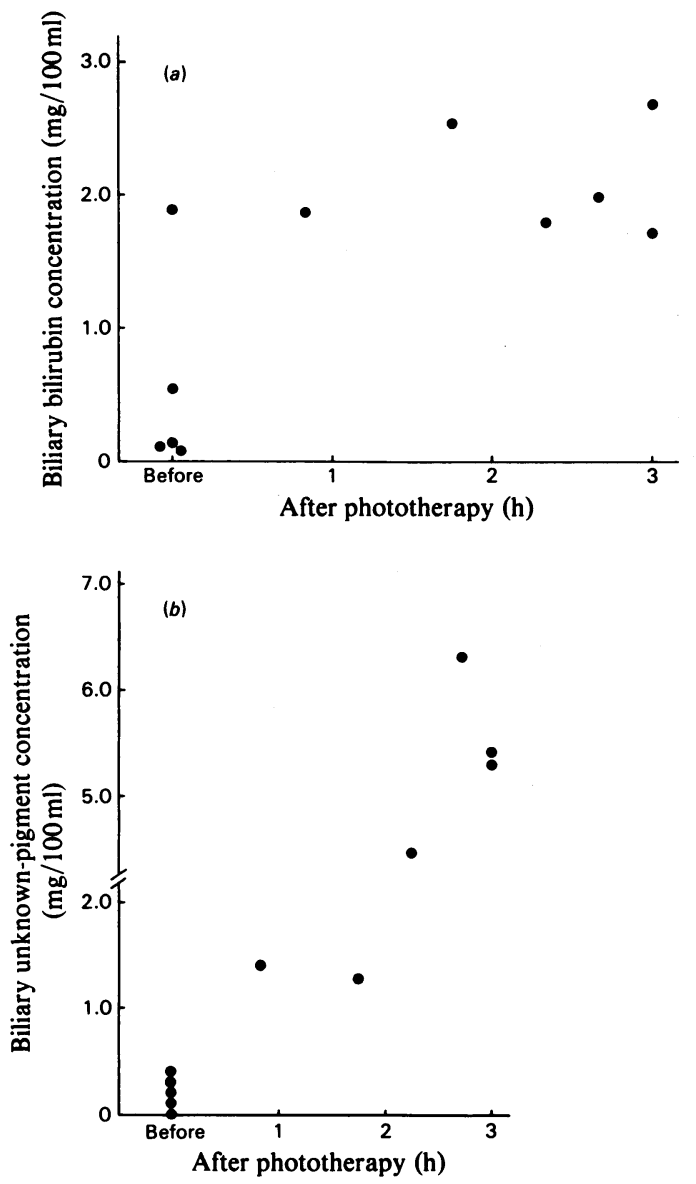

Fig. 4. Effect of phototherapy on the unconjugated bilirubin concentration $(a)$ and on the unknown-pigment concentration (b) in the duodenal aspirate 


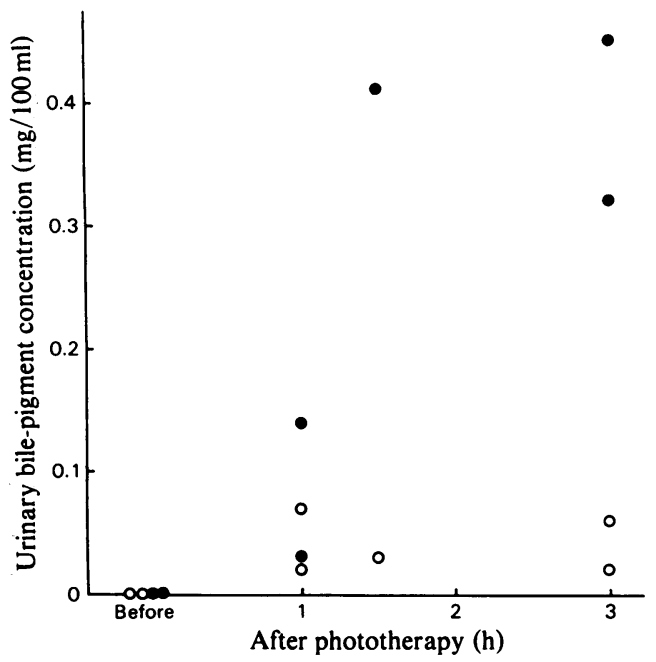

Fig. 5. Effect of phototherapy on the unconjugatedbilirubin and unknown-pigment concentrations in the urine

O, Urinary unconjugated-bilirubin concentration;

๑, urinary unknown-pigment concentration.

absorbing substance described previously (Onishi et al., 1969, 1971). The fact that the existence of photobilirubin is confirmed only in plasma and that, in contrast, only unconjugated bilirubin-IX $\alpha$ is detected in the bile suggests that the $E$-configuration is preserved during passage from the presumed site of formation near the skin surface, through the plasma and hepatocyte, to excretion in the bile. Moreover, photobilirubin in serum and unconjugated bilirubin in bile have been detected not only in treated infants, but also in all jaundiced babies, so far analysed. In bilirubin metabolism of neonatal jaundice, the excretory pathway through photobilirubin may be physiological. Therefore, the major mechanism of bilirubin metabolism during phototherapy may be anaerobic photoisomerization, as was suggested previously (McDonagh, 1976; Lightner, 1977). But it is an open question whether photobilirubin reverts to bilirubin once it has been excreted into bile. As biliary and urinary excretion of considerable amounts of the unknown pigment is observed, the significance of this pigment during phototherapy requires elucidation. It is possible that this pigment is derived from photobilirubin and is the same substance as photo-
bilirubin-IIA and -IIB described by Stoll et al. (1979).

We are grateful to Dr. A. F. McDonagh for his personal instruction and for his many helpful suggestions. This work was supported by Research Grant no. 487097 from the Ministry of Education of Japan. We thank Emeritus Professor J. Ogawa and Professor Y. Wada for encouragement.

\section{References}

Bonnett, R., Davies, J. E. \& Hursthouse, M. B. (1976) Nature (London) 262, 326-328

Bonnett, R., Davies, J. E., Hursthouse, M. B. \& Sheldrick, G. M. (1978) Proc. R. Soc. London Ser. B 202, 249-268

Lightner, D. A. (1977) Photochem. Photobiol. 26, $427-436$

Lightner, D. A., Wooldridge, T. A. \& McDonagh, A. F. (1979a) Biochem. Biophys. Res. Commun. 86, 235243

Lightner, D. A., Wooldridge, T. A. \& McDonagh, A. F. (1979b) Proc. Natl. Acad. Sci. U.S.A. 76, 29-32

Lund, H. T. \& Jacobsen, J. (1972) Acta Pediatr. Scand. 61, 693-696

McDonagh, A. F. (1976) Biochem. Soc. Trans. 4, 219-222

McDonagh, A. F. \& Ramonas, L. M. (1978) Science 201, 829-831

McDonagh, A. F., Lightner, D. A. \& Wooldridge, T. A. (1979) J. Chem. Soc. Chem. Commun. 110-112

Onishi, S., Shimidzu, K. \& Yamakawa, T. (1969) Shonika Rinsho 22, 138-150

Onishi, S., Yamakawa, T. \& Ogawa, J. (1971) Int. Cong. Pediatr. XIIIth, vol. 1, Perinatology, pp. 373-379

Onishi, S., Isobe, K., Itoh, S., Kawade, N., Sakakibara, J., Sugiyama, S., Mizutani, M. \& Lee, J. (1979a) Shonika Rinsho 32, 867-882

Onishi, S., Itoh, S., Kawade, N., Isobe, K. \& Sugiyama, S. (1979b) Biochem. Biophys. Res. Commun. 90, 890-896

Onishi, S., Itoh, S., Kawade, N., Isobe, K. \& Sugiyama, S. (1980a) Biochem. J. 185, 281-284

Onishi, S., Itoh, S., Kawade, N., Isobe, K. \& Sugiyama, S. (1980b) J. Chromatogr. 182, 105-109

Onishi, S., Kawade, N., Itoh, S., Isobe, K. \& Sugiyama, S. (1980c) Biochem. J. 190, 527-532

Ostrow, J. D. (1971) J. Clin. Invest. 50, 707-718

Ostrow, J. D., Berry, C. S. \& Zarembo, J. E. (1974) in Phototherapy in the Newborn - An Overview (Odell, G. B., Schaffer, R. \& Simopoulos, A. B., eds.), pp. 7492, National Academy of Sciences, Washington, DC

Seligman, J. W. (1977) Pediatr. Clin. North Am. 24, 509-527

Stoll, M. S., Zenone, E. A., Ostrow, J. D. \& Zarembo, J. E. (1979) Biochem. J. 183, 139-146 